\title{
Magnetic Resonance Radiofrequency Pulse Design by Optimal Control ${ }^{\star}$
}

\author{
Armin Rund* Christoph S. Aigner ${ }^{* *}$ Karl Kunisch*,*** \\ Rudolf Stollberger ${ }^{* *, * * * *}$ \\ * Institute for Mathematics and Scientific Computing, University of \\ Graz, Heinrichstr. 36, 8010 Graz, Austria and BioTechMed-Graz, 8010 \\ Graz, Austria (e-mail: armin.rund@uni-graz.at). \\ ** Institute of Medical Engineering, Graz University of Technology, \\ Stremayrgasse 16/III, 8010 Graz, Austria. \\ *** Johann Radon Institute for Computational and Applied \\ Mathematics (RICAM), Austrian Academy of Sciences, \\ Altenbergstraße 69, 4040 Linz, Austria. \\ **** BioTechMed-Graz, 8010 Graz, Austria.
}

\section{INTRODUCTION}

Progress in magnetic resonance imaging (MRI) often relies on optimal design of radiofrequency (RF) pulses. The aim is usually to perform a specific excitation/refocusing of the nuclear magnetization vector, whose dynamics can be predicted by the Bloch equations. However, RF pulse design is not a trivial task and different approaches were proposed, see e.g. Conolly et al. (1986), Pauly et al. (1991), Rund et al. (2017).

Here, a flexible optimal control model based on the Bloch equations is introduced. The main technical restrictions of a MR scanner hardware are modeled as inequality constraints. An optimization method based on a combined semismooth Newton/quasi-Newton method, penalty methods and trust-region globalization is built. The method is extended to time-optimal control for designing minimumduration RF pulses. The methods are tested in realistic numerical experiments. The optimized RF pulses are validated in phantom experiments on a $3 \mathrm{~T}$ MR scanner.

\section{MODELING}

Broad clinical imaging applications are based on sliceselective excitation (single-slice or recently simultaneous multislice acquisition Barth et al. (2016)), where data for one or multiple slices are collected at each acquisition. The modeling is here usually done in $1 \mathrm{D}$, where a slice is defined as covering an interval in $z$-direction and extending along the other two directions $x$ and $y$. The dynamics of the nuclear magnetization vector $\mathbf{M}$ is then modeled using the Bloch equation (without relaxation and in the rotating frame of reference)

(1) $\dot{\mathbf{M}}(t, z)=\gamma \mathbf{B}(t, z) \times \mathbf{M}(t, z), \quad \mathbf{M}(0, z)=\mathbf{M}_{0}(z)$ with spatial position $z \in(0, L), L>0$, time $t \in I=(0, T)$, and $T>0$. The initial condition $\mathbf{M}_{0}(z)$ is typically the steady state, i.e. aligned with the strong constant external

\footnotetext{
* This work is partially funded by the Austrian Science Fund (FWF) in the context of project "SFB F32-N18" (Mathematical Optimization and Applications in Biomedical Sciences). Partial support from BioTechMed-Graz is gratefully acknowledged.
}

magnetic field (in $z$-direction) $\mathbf{M}_{0}(z)=M_{0}(z)(0,0,1)^{T}$ with equilibrium magnetization $M_{0}(z)>0$. The MR hardware allows a user-defined input of amplitudes of different magnetic fields, which are the RF pulse $(u(t), v(t))$ and the amplitude of the slice-selective gradient $w(t)$. These magnetic fields superpose to $\mathbf{B}(t, z)=(u(t), v(t), w(t) z)$.

\section{MODELING OF THE OPTIMAL CONTROL PROBLEM}

RF pulse design typically aims at designing the timedependent amplitudes $\mathbf{u}(t)=(u(t), v(t), w(t))$ in order to optimize the competing goals of a minimum specific absorption rate (SAR), a minimum duration $T$, and an optimal slice profile accuracy at the terminal time $T$. The first two goals give rise to two objective formulations. For single transmit MRI the SAR is proportional to the power of the RF pulse, which leads to the objective

$$
\min _{\mathbf{u}(t)} J_{\mathbf{e}}=\frac{1}{2}\|r\|_{L^{2}(I)}^{2}+\frac{\alpha_{w}}{2}\|w\|_{L^{2}(I)}^{2}
$$

with $u=r \cos (\varphi), v=r \sin (\varphi)$ and $u^{2}+v^{2}=r^{2}$. Alternatively, we minimize for the duration

$$
\min _{\mathbf{u}(t), T>0} J_{\mathbf{t}}=T+\frac{\alpha_{r}}{2}\|r\|_{L^{2}(I)}^{2}+\frac{\alpha_{w}}{2}\|w\|_{L^{2}(I)}^{2} .
$$

In both cases, $\alpha_{w}, \alpha_{r}>0$ denote regularization parameters. The third goal (best approximation of a spacedependent desired magnetization pattern in $L^{2}$ or $L^{\infty}$ ) is posed as nonlinear state constraint

$$
\mathbf{g}(\mathbf{M}(T, z)) \leq 0, \quad \forall z \in(0, L) .
$$

Technical constraints on the scanner hardware are modeled as box constraints

$$
0 \leq r \leq r_{\max }, \quad|w| \leq w_{\max }
$$

as well as slew rate constraints $\dot{w} \leq s_{\max }$ and a power constraint for the time-optimal setting $\|r\|^{2} \leq P_{\max }$. Therein, $r_{\max }, w_{\max }, s_{\max }, P_{\max }>0$.

\section{METHODS}

A piecewise constant discretization for the control $\mathbf{u}(t)$ is applied. Then, (1) can be solved exactly using complex 
rotation matrices (Cayley-Klein formalism, see Pauly et al. (1991)). For fixed $T$ the control constraints (5) are treated with semismooth Newton techniques using the reformulation of the first-order necessary conditions based on Robinson's normal map, see Pieper (2015). Here, we introduce a combined semismooth Newton / quasi-Newton method, were the smooth part of the second derivative is replaced by a BFGS approximation, which avoids tangent/adjointfor-Hessian solves. The state constraints are treated with penalty methods. Adjoint based exact discrete derivatives are formed for the first derivatives. The Newton-type method is embedded into a trust-region framework using Steihaug-cg, see Steihaug (1983). The method is extended to time-optimal control by using a bilevel method, where the free terminal time is reduced in an outer loop. Here, a heuristics for global optimization is applied to exclude noncompetitive local optimizers. The methods are programmed in MATLAB, the core is parallelized in $\mathrm{C}$ using OpenMP.

\section{NUMERICAL EXPERIMENTS}

The optimization method is tested on a set of realistic examples from Grissom et al. (2016) with different number/thickness/position of the slices, and for different constraint values. A fine resolution in space and time is needed (up to 20000 points each) especially for thin slices. For measurements the temporal resolution is given by the minimum gradient raster time of the MR scanner device $(10 \mu \mathrm{s})$. The optimization runs are initialized with an existing (non-iterative) method of RF pulse design for simultaneous multislice acquisition.

In the time-fixed setting, the power of the RF pulse is reduced by approximately $50 \%$ compared to the initial pulse. In the time-optimal case, the pulse duration $T$ can be reduced by $70-90 \%$ depending on the example. Two typical time-optimal controls are depicted in Figure 1. As can be seen, both $u$ and $\dot{g}$ are bang-bang solutions everywhere but at around the two peaks of $w$. Here, other inequality constraints are active that prevent a further time reduction. In examples with tighter SAR or profile constraints, the percentage of bang-bang arcs is decreased significantly. The optimized RF pulses are implemented on a $3 T$ MR scanner and the measurements are compared to the simulations.

\section{CONCLUSION}

A tailored design of RF pulses for clinical applications is important for future directions in MRI. With the presented approach extremly short RF pulses can be generated that allow to speed up MR acquisitions significantly. In a separate work the time-optimal RF pulses were incorporated in a clinical MRI sequence resulting in a fast high-resolution full-head coverage (in 70s), see Aigner et al. (2017). The extension to parallel-transmit MRI will be investigated in the future.

\section{REFERENCES}

Aigner, C.S., Rund, A., Bilgic, B., Gagoski, B., Setsompop, K., Kunisch, K., and Stollberger, R. (2017). Application of time-optimal simultaneous multi-slice refocusing to TSE/RARE. In Proc. ISMRM 25, 3843.
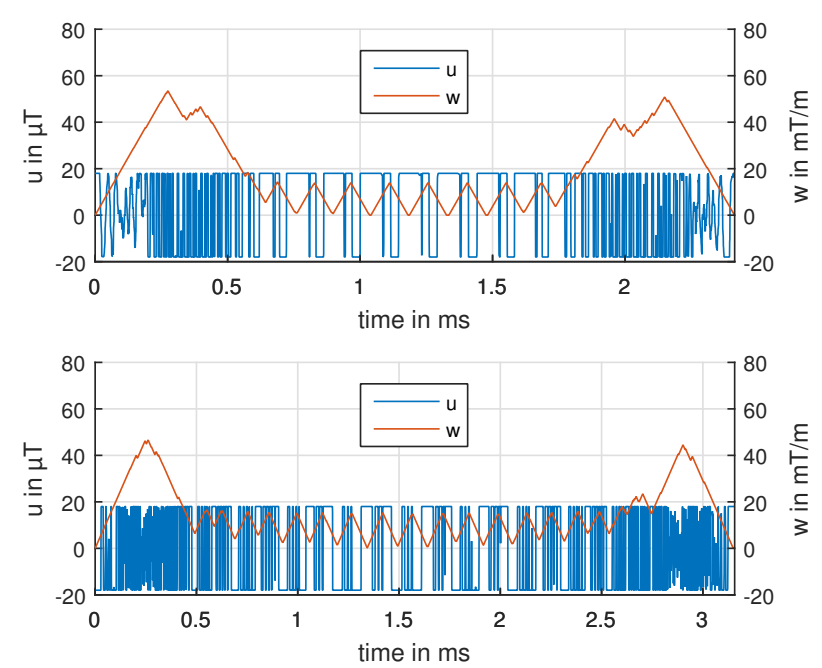

Fig. 1. Time-optimal controls $(u, w)$ using $J_{t}$ for two typical examples, a SMS refocusing pulse for a diffusion (top) and a turbo-spin echo sequence (bottom). The $\mathrm{RF}$ pulses are real-valued $(v=0)$ in these examples.

Barth, M., Breuer, F., Koopmans, P., Norris, D., and Poser, B. (2016). Simultaneous multislice (SMS) imaging techniques. Magnetic Resonance in Medicine, 81, 63-81.

Conolly, S., Nishimura, D., and Macovski, A. (1986). Optimal control solutions to the magnetic resonance selective excitation problem. IEEE Transactions on Medical Imaging, MI-5(2), 106-115.

Grissom, W.A., Setsompop, K., Hurley, S.A., Tsao, J., Velikina, J.V., and Samsonov, A.A. (2016). Advancing $\mathrm{RF}$ pulse design using an open-competition format: Report from the 2015 ISMRM challenge. Magnetic Resonance in Medicine, 1-10.

Pauly, J., Le Roux, P., Nishimura, D., and Macovski, A. (1991). Parameter relations for the Shinnar-Le Roux selective excitation pulse design algorithm. IEEE Transactions on Medical Imaging, 10(1), 53-65.

Pieper, K. (2015). Finite element discretization and efficient numerical solution of elliptic and parabolic sparse control problems. $\mathrm{PhD}$ Dissertation, Technische Universität München.

Rund, A., Aigner, C., Kunisch, K., and Stollberger, R. (2017). Magnetic resonance RF pulse design by optimal control with physical constraints. IEEE Transactions on Medical Imaging, https://doi.org/10.1109/TMI.2017.2758391.

Steihaug, T. (1983). The conjugate gradient method and trust regions in large scale optimization. SIAM Journal on Numerical Analysis, 20, 626-637. 